\title{
Retos de la pedagogía en el siglo XXI
}

Adelmo Sabogal Padilla ${ }^{1}$

\section{Resumen}

La pedagogía es un llamado a ver la realidad como un gran sistema, que debe ser analizado desde una perspectiva educativa en beneficio del desarrollo social. De esta manera, es importante determinar la pedagogía como el saber, que permitirá hacer este análisis, en donde prevalecerá la articulación entre teoría y práctica. Es necesario entonces definir cuáles son los conceptos claves del saber pedagógico. Bedoya (2008), afirma que el concepto de formación, es el principal de ellos y que alrededor de él, se pueden seguir definiendo otros, soportado siempre en el marco de la investigación.

Palabras clave: educación a distancia, pedagogía, reeducación. 


\title{
Challenges of teaching in the XXI century
}

\begin{abstract}
Pedagogy is a call to perceive reality as a large system that must be analyzed from an educational perspective for social development. It is important to define pedagogy as part of knowledge in order to allow this type of analysis where prevails the articulation between theory and practice. It is therefore necessary to define what the key concepts of pedagogical knowledge are. Bedoya (2008), states that the concept of training, is the main one and that other concepts may come from it to help define other class of concepts supported by research.
\end{abstract}

Keywords: pedagogy, reeducation, distance education.

Recibido: $12 / 08 / 2014$

Aceptado: 10/12/2014

\section{Introducción}

El proceso de formación busca la estructuración del conocimiento, que se caracteriza por diversos obstáculos como el del sentido común (científico) y el del conocimiento sensible o la experiencia básica. Se parte de una práctica, de una situación concreta y se llega a través de la aplicación de diversos procedimientos (metodología) a la producción de una teoría, es decir a la explicación de un problema planteado a partir de dicha situación concreta. Toda teoría se apoya y fundamenta en una práctica y esta debe validar y permitir aplicar determinados procedimientos o verificación de aquella (Bedoya, 2008).

De los elementos mencionados por Bedoya (2008), sobre el proceso de formación, es necesario destacar: la estructuración del conocimiento, el sentido común, el conocimiento sensible, la práctica, la aplicación de procedimientos, los problemas, la solución de problemas y la validación o verificación de procedimiento. De esta manera surge la necesidad de un saber que articule coherentemente, con sentido, desde referentes claros y propósitos contextuales, los anteriores elementos. El saber pedagógico emerge en este difícil y actual descuidado escenario. Actualmente, refuerza Bedoya (2008), se parte de la superación del empirismo y el positivismo que ha permitido a la pedagogía abrirse espacios de diálogo y confrontación entre el realismo y el racionalismo.

La pregunta por el campo específico en el que debe considerarse la pedagogía, tiene respuesta y obedece al campo de la educación. Todo lo que esté relacionado a ella, debe haberse planeado, diseñado, ejecutado y evaluado en el marco 
del saber pedagógico. Actualmente en el contexto colombiano escuchamos programas como pedagogía reeducativa, pedagogía infantil, pedagogía social comunitaria, entre las más mencionadas, que no constituyen un campo específico si no una población específica o contexto al que se le ha dado profundidad por sus características e impacto generalmente social. La reflexión en este caso, es la vinculación de la pedagogía en licenciaturas como matemáticas, ciencias sociales, ciencias básicas, idiomas, y demás, o en programas como psicología, derecho, medicina, ingenierías, sociología, antropología, administración, entre las más seleccionadas por los estudiantes, o su papel en modalidades presenciales o a distancia. En todos los casos anteriores, que pertenecen al campo de la educación, el saber pedagógico debe rescatarse como el planificador, diseñador, ejecutor y evaluador, que clarifique la articulación entre los elementos mencionados anteriormente por Bedoya

\section{Análisis y discusión}

En la actualidad en el escenario educativo, se ha generado el fenómeno de las transversalidades. Proyectos transversales, competencias y habilidades desarrolladas de manera transversal, currículos transversales, evaluaciones transversales, por mencionar algunas, que son una necesidad. Las instituciones de educación en cualquier nivel o modalidad, han dado respuesta a lo desconocido desde la palabra transversalidad; allí se encuentra todo y no se encuentra nada. El gran ausente en este fenómeno es el saber pedagógico, que requiere de trabajo cooperativo, debate académico, trazado de acuerdos y referentes claros para establecer diferencias y semejanzas, en esta entramado de la transversalidad.

La política nacional, ha permitido que cada institución divague pedagógicamente, a veces por conveniencia, para cumplir con factores externos de calidad, sin establecer estructuras internas de articulación claras en su proceso de formación. Formar a profesionales en pedagogía, es formar en el pensamiento reflexivo, en teorías, modelos, enfoques y procesos educativos de aprendizaje; en la estructuración de mesas de trabajo, en la mediación de debates académicos, en el liderazgo institucional y en la interpretación, argumentación y generación de políticas públicas a favor de la educación. Las instituciones requieren en la educación básica y media de: orientadores, profesores, coordinadores, rectores, administrativos, estudiantes padres de familia, políticas públicas y demás, pero requieren de pedagogos. Las instituciones de educación superior requieren de: rectorías, vicerrectorías, gerentes, decanos, secretarios académicos, profesores, auxiliares, estudiantes y demás, pero requieren de pedagogos. Si pensamos en las instituciones creadas por el Estado o por empresas privadas dedicadas a la educación, debemos pensar en el escenario de la pedagogía. En cualquiera de los casos anteriores, la vinculación de profesionales, especialistas, magísteres o doctores, que han pensado su vida académica en el campo de la pedagogía, empezará a dar opciones eficientes de gestión académica, encaminadas a los 
procesos pedagógicos, desarrollo curricular, sistemas de evaluación del proceso de aprendizaje, seguimiento estudiantil, modelos y enfoques pedagógicos y sobre todo una respuesta a la lógica de la transversalidad. Desde esta mirada, podríamos llegar a estructurar licenciaturas en pedagogía y diseño formativo, pedagogía y calidad educativa, pedagogía y educación de la virtualidad, pedagogía e investigación social, pedagogía y transversalidad, pedagogía, interculturalidad e inclusión, pedagogía y currículo, y pedagogía comunitaria entre otras que hoy requiere nuestro contexto social.

El contexto presentado anteriormente, es uno de los derroteros de la pedagogía en el siglo XXI. Las poblaciones que se han focalizado y que acertadamente desde la pedagogía han iniciado su reflexión, están en la necesidad de recuperar su historia e iniciar un trabajo en RED, que les permita visibilizar su trabajo y hacer propuestas concretas que tengan incidencia en las políticas públicas.

Hoy uno de los escenarios con más necesidad del pedagogo, es en el liderazgo de equipos de diseño formativo, didáctico o instruccional. En estos equipos, el pedagogo cumple el papel articulador del proceso de diseño, él es el eje que orienta y facilita el proceso, en el diseño y mantiene la articulación con otros sistemas de gestión. Conoce, no solo los contenidos de los siguientes elementos, sino esencialmente, las posibilidades de relación y articulación entre ellos: (a) teorías de aprendizaje, (b) modelos pedagógicos, (c) modelos de diseño instruccional, (d) enfoques de aprendizaje y (e) sistemas de evaluación. Sus capacidades creativas e innovadoras deben ser puestas en juego en la elaboración de guías, guiones o material que oriente el proceso del equipo. En esta estructura existe un propósito claro de rescatar la labor del pedagogo, debido al análisis constante de elementos propios de la educación tales como la didáctica, el diseño, el desarrollo y la evaluación del aprendizaje. Al respecto, Herrán (2012), afirma que las instituciones educativas han causado un desconocimiento de los aportes de los pedagogos y la pedagogía, inclusive confundiéndola con la filosofía, la política, la psicología entre otras. En el contexto colombiano añadiríamos que inclusive asociándola como un saber intrínseco en cualquier programa de licenciatura. En consecuencia, hoy y debido a la necesidad del diseño instruccional o formativo en procesos educativos mediados o no por plataformas, valdría la pena reposicionar la labor del pedagogo-investigador e impulsar el desarrollo de nuevos programas profesionales en el campo. Ahora bien, esta necesidad que ha sido potenciada por la educación virtualizada o en la virtualidad, debe necesariamente impulsar un proceso de mejora en los procedimientos de diseño, desarrollo, gestión y evaluación en la educación cara a cara.

\section{La pedagogía reeducativa}

En otro campo como el de la pedagogía reeducativa, hay que recordar históricamente, que desde el 12 de abril de 1889 en Masamagrell pueblo de la 
provincia de Valencia en España y que por idea del Obispo Fray Luis Amigó y Ferrer, sacerdote Franciscano Capuchino, se funda la congregación de Religiosos Terciarios Capuchinos de Nuestra Señora de Los Dolores, que fue conformada por una joven comunidad religiosa, la cual llevaba una vida consagrada como misión vocacional, y asumió una obra social en la que a los religiosos se les encomendó la organización de instituciones carcelarias (González, 1983).

El término reeducación se fue acuñando con la creación de establecimientos correccionales para jóvenes en el siglo XIX, que inicialmente no querían utilizar la coacción como medio para el restablecimiento de la conducta. A partir de tres grandes pilares propuestos por el Padre Luis Amigó: (a) la piedad, (b) el estudio y (c) el trabajo, se desarrolla la primera propuesta de reeducación. La estructura del proceso se despliega en varias etapas: reflexión, esperanza, perseverancia y confianza; buscando con esto la inserción de estos jóvenes a la cultura, a la sociedad, al mundo (Bohórquez, 2009).

La intervención de los Religiosos Terciarios Capuchinos en las instituciones de reeducación parte de una primera experiencia empírica basada en el afecto, la credibilidad en el otro, la paciencia y la espera; posteriormente inician un proceso de cientificidad, en el que el sujeto no es mirado sólo desde la óptica de desvalido, sino como sujeto capaz de transformar su vida, a través de una metodología psicopedagógica que superaba la mera pedagogía del amor (Bohórquez, 2009). De este proceso surgen estudios centrados en lo biopsicosocial, psicológico, pedagógico, estadístico y en relación a los derechos del menor, apoyados en la investigación científica, natural y mixta (Bohórquez, 2009).

En Colombia se inicia con éxito la materialización del proyecto de formación superior en el campo de la Licenciatura en Pedagogía Reeducativa, que recupera la historia comprimida en los párrafos anteriores, pero la reflexión actual de la cual somos responsables, debe centrarse en los siguientes campos de proyección: aportes del licenciado en pedagogía reeducativa, objeto de estudio actual, e investigación.

Inicialmente, el aporte del Licenciado en Pedagogía Reeducativa no debe desaparecer. Como pedagogo está en la responsabilidad de liderar los discursos centrados en el aprendizaje, la transversalidad, la calidad educativa y la coherencia epistemológica de la planeación y acción educativa. Como reeducador, debe mantener el liderazgo de los equipos de trabajo en los centros de menores infractores, pero debe generar expectativa en las instituciones de educación básica, media o superior, inicialmente logrando posicionamiento profesional académico y administrativo, para proponer políticas públicas que posicionen la pedagogía como necesidad educativa obligatoria en toda institución. También, debe encaminar su quehacer a los campos de la interculturalidad y la inclusión, en donde los proyectos sociales demandan, estrategias de intervención orientadas desde la pedagogía. Finalmente como profesional, debe mantenerse en constante 
capacitación, lo cual permita visibilizar su pensamiento y materializar sus propuestas, en el escenario académico.

En segundo lugar, si reflexionamos sobre el objeto de estudio del pedagogo reeducador, me referiría nuevamente al campo de la transversalidad, que no siendo tan fácil de comprender en su metodología de apropiación, si es evidente en su necesidad cotidiana. Como se mencionó inicialmente, este es un objeto de estudio del pedagogo en general, pero el reeducador debe matizar su reflexión en tres elementos: la inclusión, la interculturalidad y la reintegración ciudadana.

Finalmente, en el camino de la investigación el cual considero un gran aporte del currículo establecido para los licenciados en Pedagogía Reeducativa, es inminente la alineación de este esfuerzo desde la claridad conceptual y metodológica en campos como la investigación acción, la investigación acción participativa, la investigación etnográfica, la investigación narrativa, la investigación en teoría fundada y la investigación formativa, desde el marco de la investigación cualitativa. De la misma manera la continuidad de estos proyectos en campos de formación pos gradual y su socialización en espacios académicos nacionales e internacionales, deben generar la discusión colectiva y la iniciativa por el posicionamiento de la pedagogía como necesidad institucionalizada a nivel nacional.

\section{Referencias bibliográficas}

Bedoya, J. 2008. Pedagogía ¿Enseñar a pensar? Bogotá: ECOE.

Bohórquez, O. 2009. Revista Poiésis FUNLAM No 17 (Junio de 2009). Recuperado de http://www.funlam.edu.co/revistas/index.php/poiesis/article/view/198

Creswell , J. 2006. Educational Research: Planning, Conducting, and Evaluating Quantitative and Qualitative Research, third edition. Boston: University of Nebraska / Pearson.

Egido Gálvez, I. 2012. El pedagogo en los centros de secundaria. En: A. de La Herrán (coord.). Apuntes de pedagogía. Recuperado en: http://www.uam.es/personal_pdi/ fprofesorado/agustind/textos/apuntespedagogia10.pdf. Consultado el 28 de noviembre de 2014 .

González, F. 1983. Biografía de Fray Luis Amigó y Ferrer. Valencia: Talleres gráficos Nacher. 